\title{
Proper motions and CCD-photometry of stars in the region of the open cluster NGC 1513
}

\author{
V. N. Frolov ${ }^{1}$, E. G. Jilinski ${ }^{1,2}$, J. K. Ananjevskaja ${ }^{1}$, E. V. Poljakov ${ }^{1}$, N. M. Bronnikova ${ }^{1}$, and D. L. Gorshanov ${ }^{1}$ \\ 1 The Main Astronomical Observatory, Pulkovo, St. Petersburg, Russia \\ ${ }^{2}$ Laboratório Nacional de Computação Científica / MCT, Petrópolis, RJ, Brazil
}

Received 3 July 2002 / Accepted 10 September 2002

\begin{abstract}
The results of astrometric and photometric investigations of the poorly studied open cluster NGC 1513 are presented. The proper motions of 333 stars with a root-mean-square error of 1.9 mas $\mathrm{yr}^{-1}$ were obtained by means of the automated measuring complex "Fantasy". Eight astrometric plates covering the time interval of 101 years were measured and a total of 141 astrometric cluster members identified. BV CCD-photometry was obtained for stars in an area $17^{\prime} \times 17^{\prime}$ centered on the cluster. Altogether 33 stars were considered to be cluster members with high reliability by two criteria. The estimated age of NGC 1513 is $2.54 \times 10^{8}$ years $^{\star}$.
\end{abstract}

Key words. open clusters and associations: general - open clusters and associations: individual: NGC 1513

\section{Introduction}

The open cluster NGC 1513 is located in the Perseus constellation. Its equatorial and galactic coordinates are:

$\alpha=4^{\mathrm{h}} 09^{\mathrm{m}} 98^{\mathrm{s}}, \delta=+49^{\circ} 31^{\prime} ; \ell=152^{\circ} .6, b=-1.57$ (2000.0).

In the Trümpler (1930) catalogue it is classified as II $2 \mathrm{~m}$ a moderately rich cluster with little central concentration. It was investigated astrometrically by Bronnikova (1958a) who determined the proper motions (PM) using a single pair of plates with an epoch difference of 55 years. Barhatova \& Drjahlushina (1960) published photographic and photovisual magnitudes of 49 stars from Bronnikova's (1958b) list. Del Rio \& Huestamendia (1988) (RH) obtained photoelectric $U B V$ magnitudes of 31 stars and photographic $R G U$ magnitudes for 116 stars in the cluster region. Spectra and radial velocities of stars in this area have not been investigated. As the epoch difference has increased significantly since the time of the last and only attempt to investigate proper motions of the stars in the region of the open cluster NGC 1513, we decided to study it using the material available at present.

Send offprint requests to: V. N. Frolov, The Main Astronomical Observatory, Pulkovo, St. Petersburg, Russia

e-mail: vfrol@gao.spb.ru

* Tables 2 and 3 are only available in electronic form at the CDS via anonymous ftp to cdsarc.u-strasbg.fr $(130.79 .128 .5)$ or via http://cdsweb.u-strasbg.fr/cgi-bin/qcat?J/A+A/396/125
Table 1. Astrometric plates.

\begin{tabular}{lccl}
\hline \hline Plate & $\begin{array}{c}\text { Exposure } \\
\text { (min) }\end{array}$ & Epoch & Quality \\
\hline \multicolumn{4}{c}{ Early epoch } \\
A 371 & unknown & 1899 Nov. 30 & good \\
D 451 & 16 & 1951 Feb. 7 & high \\
5011 & 26 & 1954 Nov. 25 & good \\
5017 & 26 & 1954 Nov. 30 & high \\
\multicolumn{4}{c}{ Second epoch } \\
18152 & 20 & 1999 Nov. 19 & good \\
18159 & 25 & 1999 Dec. 12 & poor \\
18170 & 30 & 2000 Feb. 2 & poor \\
18177 & 25 & 2000 Feb. 6 & good \\
\hline
\end{tabular}

\section{Astrometry}

The observational material belongs to the Normal astrograph collection of the Pulkovo observatory and dates from 1899 to 2000 (Table 1). The cluster region from the USNO-A 2.0 catalogue was used as an additional plate (epoch 1954.8). The scale of the Normal astrograph plates is $60^{\prime \prime} \mathrm{mm}^{-1}$. The positions of 383 stars in an area $60^{\prime} \times 60^{\prime}$ centered on the cluster NGC 1513 were measured. For the first time our plates were scanned by means of the automated measuring complex "Fantasy". As there is no description of the complex in English we present it in the Appendix.

The proper motions were determined by the line-method (the star positions tranferred to one system and arranged in chronological order of the epochs of observation were compared). The details of the method are presented in the paper of 
Jilinski et al. (2000). The plate 5017 with the faintest images was used as the central plate. It was orientated using 64 stars in common with stars in the "TYCHO" catalogue so that the $Y$ axis was parallel to the sky meridian. The reference stars for the reduction were selected by several approximations. Each step consists of two successive parts. At the first step all the stars of the region were considered as reference stars. For each star, graphs were considered where the epochs of the plates were plotted as abscissa and the coordinate $X$ or $Y$ of the stars as ordinates. Two straight lines were drawn by a least square fitting. Their inclinations correspond to the proper motion components $\mu_{x}$ and $\mu_{y}$ of the star. After this preliminary determination of PM, the first rough selection of cluster members was made. Those of the stars that were located on the vector point diagram (VPD) in a circle with a radius of three standard deviation from the center of the concentration of points corresponding to the cluster were considered to be members of the cluster. As a result of the first approximation a new list of reference stars was compiled according to the generally accepted rule: that they must be uniformly distributed in the studied region excluding the cluster area, have small proper motions and belong to a chosen range of $B$ magnitudes. As the $U B V$ magnitude of only a few stars were determined in the investigated area, we had to use the considerably less precise values of $B$ magnitudes of the USNO-A 2.0 catalogue. Thus 39 stars in the interval between 13.5 and 14.5 mag were selected. The linear method of reduction was used. From the second approximation the selection of cluster members was made according to Sanders method (1971) assuming that the proper motion distributions of the cluster and field stars on the VPD were bivariate Gaussian distributions, circular for the cluster $\Phi_{\mathrm{c}}\left(\mu_{x}, \mu_{y}\right)$ and elliptical for the field $\Phi_{\mathrm{f}}\left(\mu_{x}, \mu_{y}\right)$. Modelling of the resulting distribution was fulfilled by the method of maximum likelihood. The values of the parameters of the corresponding system of the non-linear equations were determined by computer iterations. All the formulas are given in Jilinski et al. (2000). The individual membership probability of a star was determined by its position on VPD and calculated by the following formula:

$P\left(\mu_{x}, \mu_{y}\right)=\frac{N_{\mathrm{c}} \Phi_{\mathrm{c}}\left(\mu_{x}, \mu_{y}\right)}{N_{\mathrm{c}} \Phi_{\mathrm{c}}\left(\mu_{x}, \mu_{y}\right)+N_{\mathrm{f}} \Phi_{\mathrm{f}}\left(\mu_{x}, \mu_{y}\right)}$

$N_{\mathrm{c}}$ - normalized number of cluster stars,

$N_{\mathrm{f}}$ - normalized number of field stars,

$\mu_{x_{i}}, \mu_{y_{i}}$ - proper motion in $x$ and $y$ for the $i$ th star.

In order to have a fast fit in the solution of the system parameters close to the real initial values of parameters were adopted. In the final calculation of probabilities the following values were used: the relative number of cluster members and field stars $N_{\mathrm{c}} / N_{\mathrm{f}}=0.5$;

centers of the distributions on the VPD:

the field $\mu_{x_{0}}=1.34, \mu_{y_{0}}=0.45$; the cluster $\mu_{x_{0}}=-0.9$, $\mu_{y_{0}}=0.44$

standard deviations: the field $\Sigma_{x}=7.0, \Sigma_{y}=5.0$; the cluster $\sigma_{x, y}=1.5$.

All the values are in mas $\mathrm{yr}^{-1}$. Each iteration ended with plotting the histogram of probabilities. Its analysis permitted an estimation of the minimal value of the probability for a star to

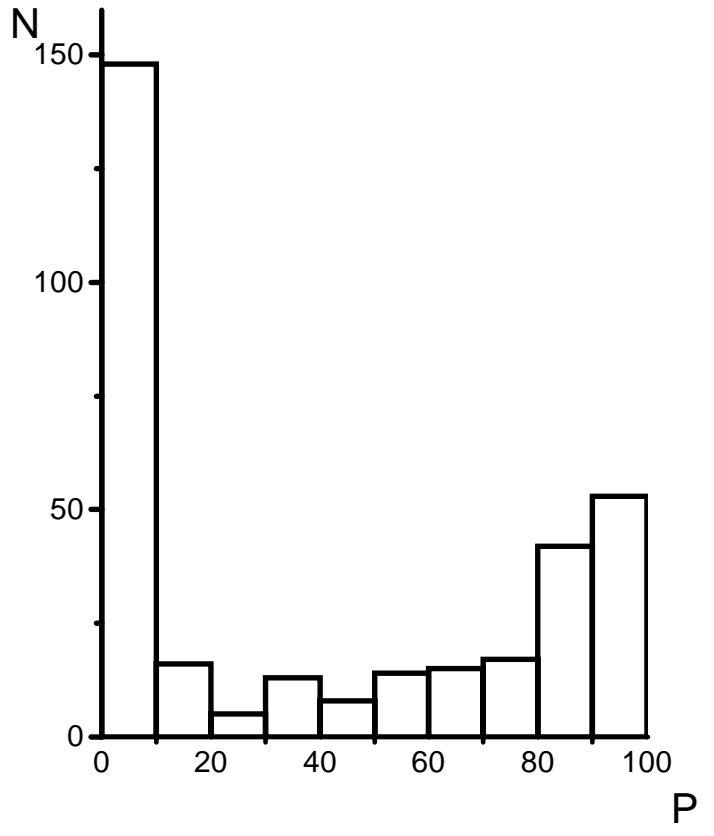

Fig. 1. The final histogram of star membership probabilities.

be considered a cluster member. The final histogram is shown in Fig. 1.

The magnitude equation (ME) or the dependence of the determined proper motions on star magnitudes was investigated at each step of the membership selection. During the first iteration the ME was derived from an investigation of the VPD for assumed field stars with proper motion components corrected for the motion of the Sun towards the solar apex. The values of these components were calculated with the help of the tables published by Zhukov (1966). Then ME was estimated by the selected cluster members anew in each iteration. The direction and inclination of the ME (for $X$-axis:

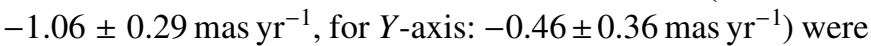
derived from the field stars in the whole interval of $B$ magnitudes from 10.5 to $15 \mathrm{mag}$. These values were used to correct those of the original catalogue. After the selection of cluster members the estimated ME was: $X:-1.95 \pm 0.42 \mathrm{mas} \mathrm{yr}^{-1}$,

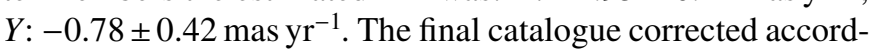
ing to these values appeared to be free of ME within the limits of the accuracy of the determination of the PM of the stars. The final VPD is given in Fig. 2.

The rms errors of the proper motion components of the stars are:

$\sigma_{x}= \pm 1.8 \operatorname{mas~yr}^{-1}, \sigma_{y}= \pm 1.9 \operatorname{mas~yr}^{-1}$.

The astrometric results are given in Table 2. Columns: 1) the number of a star according to our study (the reference stars are marked by the symbol *), 2), 3) the rectangular coordinates of a star relative to the star N $197(\mathrm{~N} 115 \mathrm{RH})$ in arcmin, 4) $B$ magnitudes from the USNO -A 2.0,5) the number of plates, 6), 7), 8), 9) PM $\mu_{x}, \mu_{y}$ and the corresponding standard errors in mas $\left.\mathrm{yr}^{-1}, 10\right)$ membership probabilities in \%, 11) "bl" marks blends, "bs" - objects whose coordinates were determined with large errors. It also contains the numbers of the stars in the BD, HD and Hipparcos catalogues. 


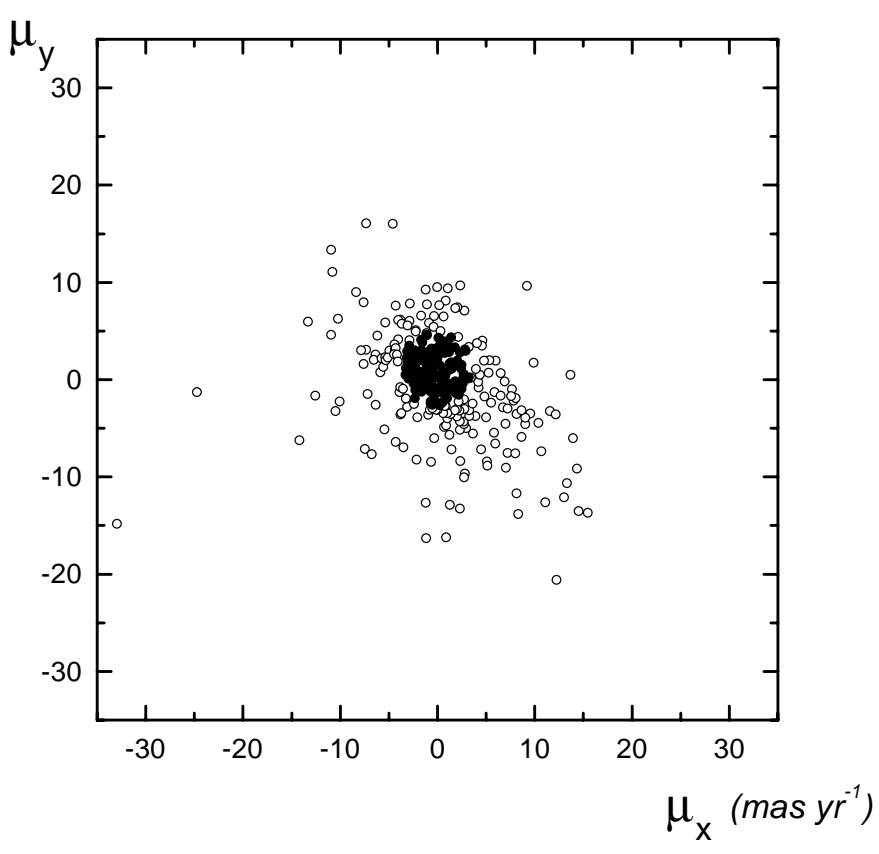

Fig. 2. The final VPD of NGC 1513.

Due to various reasons (blending, wrong identification, etc.) we were not able to determine the PM of 50 stars from the total number of 383 stars. Astrometric cluster members were considered to be stars with a membership probability $P \geq 50 \%$. The total number of such stars is 141 (the probability of 112 of them $P \geq 70 \%$ ).

\section{Photometry}

The $B$ and $V$ magnitudes of stars in the central part of the investigated area were obtained with the $320 \mathrm{~mm}$ mirror astrograph ZA-320 of the Pulkovo Observatory equipped with the CCD-receiver ST-6 with a fixed TC241 matrix. A detailed description of the telescope and its resources is given in the paper of Bekyashev et al. (1998). The area of the field determined by the scale $\left(65^{\prime \prime} \mathrm{mm}^{-1}\right)$ and the dimensions of the matrix is equal to $9.3^{\prime} \times 7^{\prime}$. Due to the small area of the field the observations of $B$ and $V$ colours were limited to the area $17^{\prime} \times 17^{\prime}$ where all stars from the RH paper are located. The $U B V$ magnitudes of 31 stars from Table 2 of their paper were used as photoelectric standards. Besides stars marked by RH numbers there are in the same field a considerable amount of stars without any numbers. They are marked by numbers beginning with " $a$ ". Some stars that are beyond the limits of the RH map were also measured and are marked by " $b$ ". The map of the cluster is given in Fig. 3 and the CCD magnitudes and colours - in Table 3 with the columns: 1) the RH number of a star, 2), 9), 14) the star number according to our study, 3), 10), 15) CCD photometry in the $V$ band, 4), 11), 16) CCD colours $(B-V), 5), 6)$ photoelectric (RH) $V$ and $(B-V), 7), 12)$ and 17$)$ notes.

The external rms errors calculated for the standard stars with zero mean deviations is $\pm 0.012 \mathrm{mag}$ in the $B$ pass-band and \pm 0.010 mag in $V$. After the reduction of the instrumental values to the standards no residual colour equestion was detected. The internal rms errors increase from the bright to the faint stars. Their mean values in the interval from $9.6 \mathrm{mag}$ to $16.37 \mathrm{mag}$ are $\sigma_{B}= \pm 0.07 \mathrm{mag}, \sigma_{V}= \pm 0.05 \mathrm{mag}$.

\section{Analysis and conclusion}

All the available $B, V$ - photometry resulted in the $V \& B-V$ (CMD) diagram given in Fig. 4. Stars with membership probability $P \geq 50 \%$ are plotted by solid circles. Obviously it is difficult to define the Main sequence (MS) due to contradictions in the published data on selective absorption in the cluster region (estimates of the mean $E_{B-V}$ vary from 0.52 mag to $0.70 \mathrm{mag}$ ). With the absence of $U$ magnitudes of the stars we had to use the ZAMS position from the RH paper. The value of the colour-excess $E_{B-V}=0.67$ was calculated from the formula $E_{B-V}=0.72 E_{G-R}-0.005\left(E_{B-V}\right)^{2}$ (Steinlin 1968) with $E_{G-R}=0.94 \mathrm{mag}$ and apparent distance modulus $\left(V-M_{V}\right)=$ 12.61 using the true modulus $\left(V-M_{V}\right)_{0}=10.60$. Stars located on the main sequence within the limits $\pm 3 \sigma$ are considered to be cluster members according to photometric criteria. In Fig. 5 the cluster members (according to photometric and astrometric criteria) are plotted as solid circles. Open circles mark probable cluster members (only by the photometric criterion) as their magnitudes are fainter than the limiting values of the Normal astrograph old plates. In Fig. 5 there are two stars with high membership probabilities at the late evolution stages: N 175: $M_{V}=-0.41 \mathrm{mag},(B-V)_{0}=0.88 \mathrm{mag}, P=93 \%$ and N 299: $M_{V}=-1.20 \mathrm{mag},(B-V)_{0}=1.08 \mathrm{mag}, P=90 \%$; ZAMS - Schmidt-Kaler (1965). The members and probable members are listed in Table 4.

The estimation of the age of the cluster is based on the set of isochrones for stars with mean masses from 0.16 to $7 M_{\odot}$ and different metallicities $(\mathrm{Z})$ published on the website of the Padova research group ${ }^{1}$ and described in the work of Girardi et al. (2000). The superposition of various isochrones on the CMD shows an almost perfect coincidence of the $2.54 \times 10^{8} \mathrm{yr}$ isochrone for $Z=0.019$ with the cluster diagram (Fig. 5). The loop that marks the ending of hydrogen burning in the core, compression of the core and hydrogen burning in the thick layer is well occupied by stars. The star No. 175 is located almost at the base of the red giant branch, and the star No. 229 - at the stage of helium burning. Corresponding to this isochrone the turn-off point of the MS is $(B-V)_{0}=-0.07 \mathrm{mag}$.

It is also interesting to note that the MS of NGC 1513 on the CMD in absolute coordinates almost completely coinsides with the MS of the nearby cluster NGC 1528. Their ages also coincide. The age and MS position of the latter were discussed in the paper by Mermilliod (1981). Are these facts accidental? It is still possible that Barhatova (1963) who treated them along with NGC 1545 cluster as a triplet related by a common origin was right. $\mathrm{RH}$ did not confirm the physical connection of NGC 1513 and NGC 1528 because of the essential difference in their distances. We think that it is worthwhile to obtain data on the membership of stars of NGC 1528 not only by the photometric diagram but also by proper motions of its stars. The Pulkovo observatory collection contains a set of negatives

\footnotetext{
${ }^{1}$ http://www. pleadi.astro.pd.it
} 
Table 4. Members and probable cluster members.

\begin{tabular}{|c|c|c|c|c|c|c|c|c|c|c|}
\hline \multicolumn{5}{|c|}{ Members } & \multicolumn{6}{|c|}{ Probable members } \\
\hline$N_{\mathrm{pm}}$ & $N_{\text {phot }}$ & $M_{V}$ & $(B-V)_{0}$ & $P$ & $N_{\text {phot }}$ & $M_{V}$ & $(B-V)_{0}$ & $N_{\text {phot }}$ & $M_{V}$ & $(B-V)_{0}$ \\
\hline 124 & $\mathrm{~b} 53$ & 1.27 & 0.09 & 59 & 4 & 2.63 & 0.26 & a 69 & 3.55 & 0.12 \\
\hline 126 & 37 & 1.68 & 0.22 & 94 & 12 & 2.30 & 0.48 & a 70 & 2.79 & 0.35 \\
\hline 127 & 38 & 0.85 & 0.14 & 95 & 15 & 2.34 & 0.31 & a 78 & 3.19 & 0.34 \\
\hline 130 & 45 & 0.40 & 0.09 & 83 & 16 & 1.99 & 0.23 & a 80 & 3.30 & 0.69 \\
\hline 132 & b12 & 0.33 & 0.09 & 88 & 17 & 2.05 & 0.12 & a 81 & 2.95 & 0.48 \\
\hline 133 & b7 & -2.18 & -0.24 & 95 & 20 & 2.05 & 0.36 & a 93 & 3.15 & 0.58 \\
\hline 159 & 48 & 1.11 & -0.02 & 90 & 23 & 2.56 & 0.27 & a 97 & 3.38 & 0.68 \\
\hline 160 & 76 & -1.15 & -0.11 & 66 & 24 & 1.74 & 0.30 & a112 & 2.56 & 0.34 \\
\hline 161 & 47 & 0.64 & -0.08 & 94 & 28 & 1.87 & 0.23 & a117 & 3.47 & 0.10 \\
\hline 162 & 49 & 0.19 & -0.15 & 77 & 29 & 2.07 & 0.30 & a123 & 3.51 & 0.59 \\
\hline 163 & 50 & -0.60 & -0.07 & 94 & 33 & 2.42 & 0.52 & a124 & 2.82 & 0.26 \\
\hline 164 & 72 & 0.54 & -0.06 & 93 & 34 & 2.03 & 0.39 & a127 & 3.05 & 0.37 \\
\hline 166 & 53 & 1.34 & 0.05 & 93 & 35 & 2.39 & 0.39 & a131 & 2.95 & 0.16 \\
\hline 167 & 54 & 1.77 & -0.01 & 80 & 39 & 2.38 & 0.01 & a133 & 2.05 & 0.37 \\
\hline 168 & 71 & -1.06 & -0.04 & 86 & 41 & 2.53 & 0.11 & a157 & 3.39 & 0.73 \\
\hline 169 & 55 & -0.64 & -0.04 & 91 & 42 & 2.88 & 0.39 & a160 & 3.29 & 0.36 \\
\hline 173 & 69 & 1.60 & -0.01 & 68 & 43 & 2.99 & 0.40 & a167 & 2.66 & 0.34 \\
\hline 174 & 58 & 1.50 & 0.19 & 94 & 51 & 2.15 & -0.07 & a168 & 2.97 & 0.45 \\
\hline 175 & 62 & -0.41 & 0.88 & 93 & 52 & 2.68 & 0.20 & a170 & 2.79 & 0.30 \\
\hline 178 & 5 & 1.76 & 0.18 & 84 & 56 & 2.89 & 0.31 & a171 & 2.50 & 0.30 \\
\hline 184 & 2 & 1.55 & 0.05 & 66 & 57 & 2.08 & 0.04 & a172 & 2.39 & 0.20 \\
\hline 185 & 1 & -0.21 & -0.04 & 62 & 59 & 2.16 & 0.04 & b 0 & 2.83 & -0.01 \\
\hline 190 & 9 & 0.77 & 0.01 & 94 & 61 & 2.21 & 0.16 & b 1 & 2.43 & 0.25 \\
\hline 194 & 18 & 1.48 & 0.21 & 91 & 70 & 1.94 & 0.03 & b 2 & 2.33 & 0.03 \\
\hline 215 & 110 & 0.49 & 0.13 & 82 & 73 & 2.55 & 0.35 & b 5 & 2.83 & 0.35 \\
\hline 216 & 113 & 1.51 & 0.27 & 90 & 74 & 2.44 & -0.07 & b 10 & 2.48 & 0.49 \\
\hline 217 & 111 & 1.50 & 0.18 & 89 & 75 & 2.75 & 0.30 & b 13 & 1.58 & 0.01 \\
\hline 219 & 106 & 1.14 & 0.17 & 56 & 77 & 2.69 & 0.20 & b 16 & 2.36 & 0.14 \\
\hline 222 & 90 & 0.66 & -0.01 & 93 & 83 & 2.51 & 0.39 & b 17 & 1.47 & 0.15 \\
\hline 229 & 88 & -1.20 & 1.08 & 90 & 84 & 2.65 & -0.04 & b 19 & 2.99 & 0.26 \\
\hline 232 & 97 & 1.82 & 0.18 & 81 & 85 & 2.50 & 0.07 & b 20 & 2.45 & 0.42 \\
\hline 234 & 101 & -0.73 & -0.09 & 52 & 89 & 1.52 & 0.04 & b 33 & 2.51 & 0.55 \\
\hline \multirow[t]{23}{*}{239} & 80 & 1.69 & 0.11 & 56 & 92 & 2.39 & 0.23 & b 42 & 2.84 & 0.37 \\
\hline & & & & & 105 & 2.41 & 0.32 & b 43 & 2.00 & 0.39 \\
\hline & & & & & 108 & 1.99 & 0.24 & b 46 & 3.09 & 0.42 \\
\hline & & & & & 109 & 2.67 & 0.18 & b 48 & 3.39 & 0.14 \\
\hline & & & & & 112 & 2.37 & 0.10 & b 49 & 2.39 & 0.17 \\
\hline & & & & & 114 & 2.24 & 0.25 & b 50 & 3.18 & 0.21 \\
\hline & & & & & a 7 & 2.02 & 0.26 & b 51 & 2.24 & 0.45 \\
\hline & & & & & a 12 & 2.06 & 0.23 & b 56 & 3.21 & 0.06 \\
\hline & & & & & a 14 & 2.13 & 0.24 & b 57 & 3.77 & 0.50 \\
\hline & & & & & a 18 & 2.11 & -0.02 & b 60 & 3.41 & 0.47 \\
\hline & & & & & a 21 & 3.78 & 0.55 & b 65 & 3.33 & 0.20 \\
\hline & & & & & a 24 & 3.51 & 0.16 & b 67 & 1.95 & -0.03 \\
\hline & & & & & a 27 & 2.63 & -0.01 & b 70 & 2.50 & 0.55 \\
\hline & & & & & a 28 & 3.02 & 0.22 & b 74 & 3.76 & 0.21 \\
\hline & & & & & a 30 & 3.45 & 0.09 & b 75 & 1.91 & 0.25 \\
\hline & & & & & a 31 & 2.88 & 0.21 & b 76 & 2.45 & 0.29 \\
\hline & & & & & a 39 & 3.03 & 0.60 & b 79 & 3.80 & 0.51 \\
\hline & & & & & a 41 & 3.68 & 0.13 & b 80 & 2.65 & 0.48 \\
\hline & & & & & a 45 & 2.63 & 0.13 & b 81 & 2.06 & 0.33 \\
\hline & & & & & a 53 & 2.00 & 0.22 & b 89 & 2.38 & 0.50 \\
\hline & & & & & a 58 & 3.34 & 0.54 & b 92 & 2.01 & 0.22 \\
\hline & & & & & a 66 & 3.55 & 0.22 & b101 & 2.97 & 0.53 \\
\hline & & & & & a 68 & 3.73 & 0.10 & & & \\
\hline
\end{tabular}




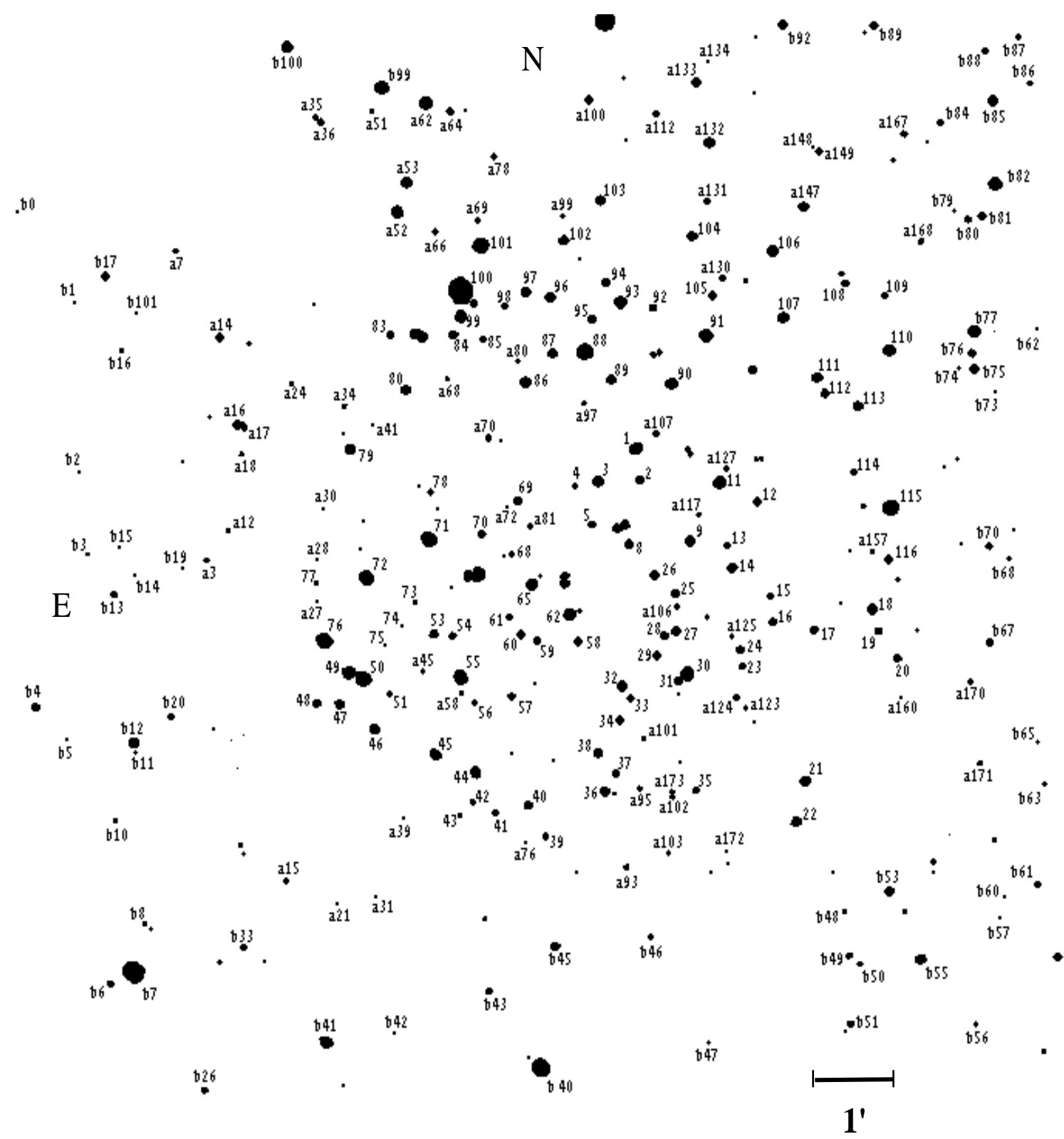

Fig. 3. CCD map of the central part of the cluster NGC 1513. The numbers of stars are given according to the photometric study: numbers without an index -RH; stars marked by the index "a" were not measured by RH; stars marked by index "b" are beyond the RH map.

taken with the Normal astrograph from 1959 to the present time.

\section{Appendix}

The automated measuring complex "Fantasy" is a programmed precision microdensitometer with capabilities determined by the software developed for it. The base of the machine is a massive $(1500 \mathrm{~kg})$ metal table with a polished surface on which a carriage moves on aerostatic bearings. The carriage is actuated by two line electric motors and its position is determined by a laser interferometer. The scanning system consists of a cathode ray tube with a program control beam and photoelectronic multiplier with the measured plate between them. The characteristics of the positioning and scanning systems are given in Table 5.
Table 5. "Fantasy" characteristics.

\begin{tabular}{ll}
\hline \hline Positioning system & Scanning system \\
Carriage operating field $370 \times 370 \mathrm{~mm}$ & View window $4 \times 4 \mathrm{~mm}$ \\
Time of positioning $4 \mathrm{~s}$ & Range of reading $20 \mathrm{kp} \mathrm{s}^{-1}$ \\
Accuracy of positioning 1 micron & Aperture 2-3 micron \\
Accuracy of position measuring $0.32 \mathrm{micron}^{-1}$ & Resolution 1 micron \\
Speed of the carriage movement $330 \mathrm{~mm} \mathrm{~s}^{-1}$ & Dynamic range 2D \\
\hline
\end{tabular}

The algorithm of position measurements developed for "Fantasy" is efficient for measurements in all ranges of stellar images on a plate, from the brightest with a diameter 600800 microns to very faint hardly discernible images consisting of separate darkened grains. In reality the secondary forms of the images are measured. These forms are constructed during 


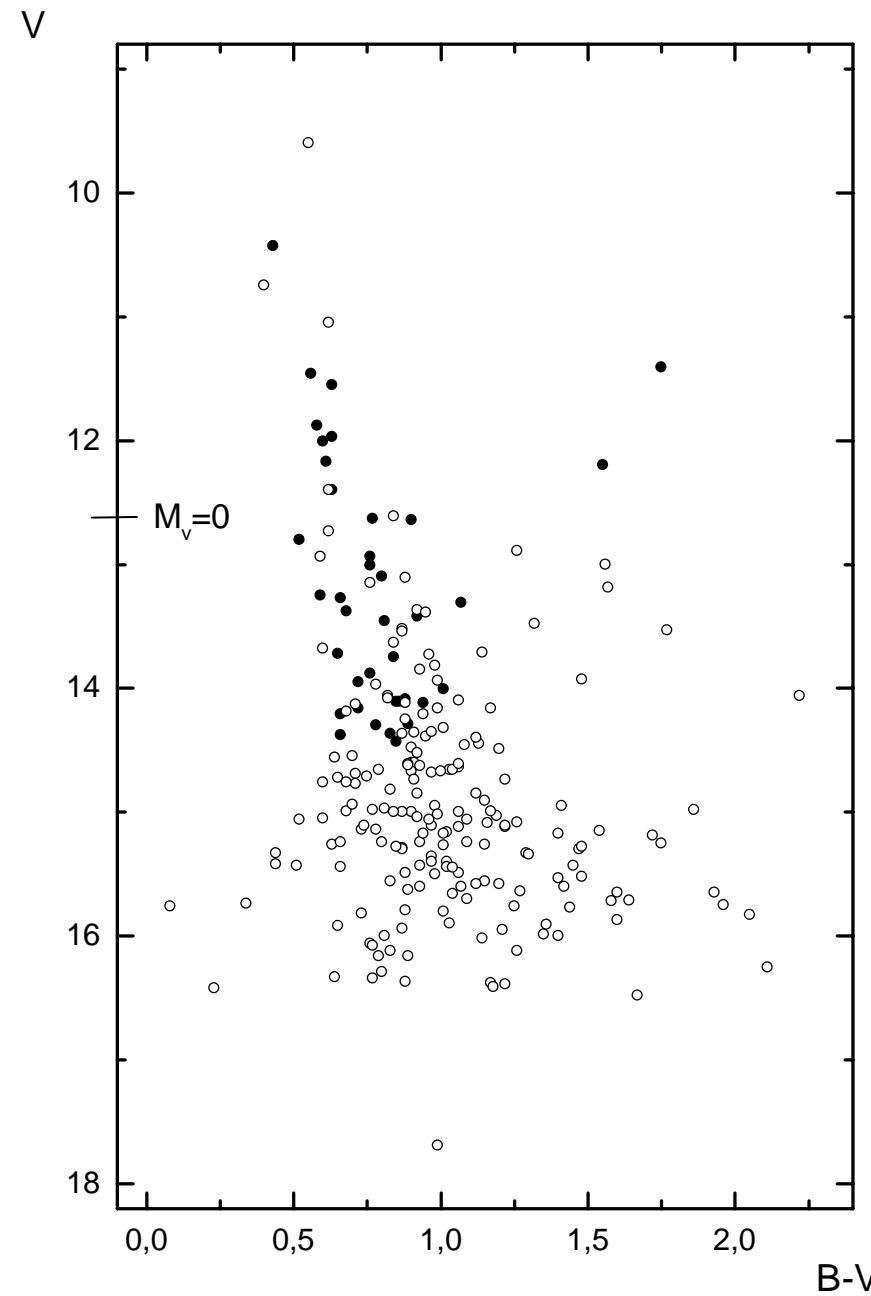

Fig. 4. CMD diagramm of the cluster NGC 1513. - stars with the membership probability $P \geqslant 50 \%$.

the processing of the images by iterative operations: filtration, contrasting and normalising. This permits the reduction of a wide range of stellar images on a plate to a set of compact objects of the regular form with centres defined as the centres of their photometric weights. A deleting operation was included in the algorithm for measurements of old plates with a Gauthier grid. The developed algorithm for position measurements has high operational and metrological characteristics. It operates accurately in conditions of considerable background density differences, low quality images and in the presence of photographic blemishes and defects. The standard error of measurement depends on the quality of the photographic material and equals $0.14-0.60$ microns for the whole range of images.

Acknowledgements. We would like to thank Dr. Z. Kadla for many useful comments on the manuscript and investigators of the Pulkovo observatory: M. Sidorov, A. Devyatkin, I. Grigorjeva and V. Kouprianov for their collaboration in the observations. E. G. Jilinski thanks FAPERJ for the financial support under the contract E-26/152.221/2000.

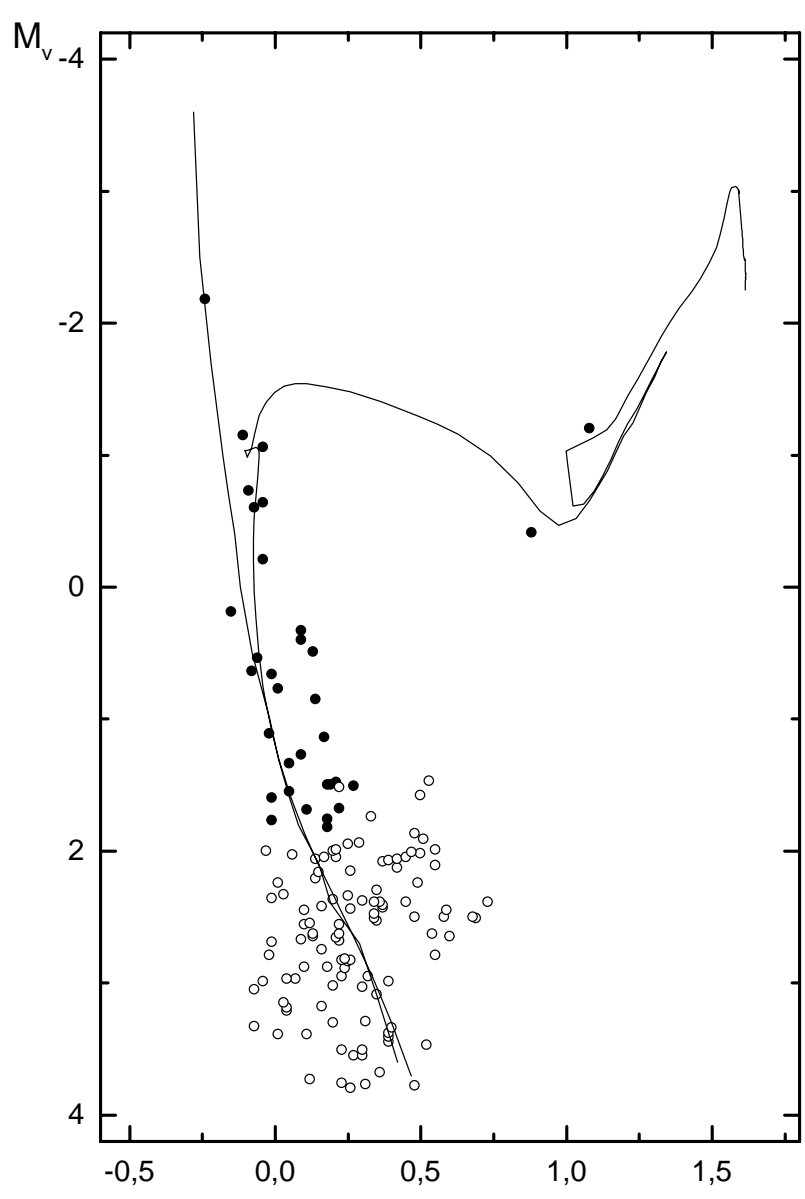

$(\mathrm{B}-\mathrm{V})_{0}$

Fig. 5. The $M_{V}$ and $(B-V)_{0}$ diagramm of all cluster members. ZAMS - Schmidt-Kaler(1965). Isochrone $-z=0.0019$, age $=2.54 \times 10^{8} \mathrm{yr}$.

\section{References}

Barhatova, K. A., \& Drjakhlushina, L. I. 1960, A Zh., 37, 332

Barhatova, K. A. 1963, Ural. Sb. 1, 30 (in Russian)

Bekyashev, R. H., Kanaev, I. I., Devyatkin, A. V., et al. 1998, Izv. Glav. Astron. Obs. Pulkovo, No. 213, 249 (in Russian)

Bronnikova, N. M. 1958a, Izv. Glav. Astron. Obs. Pulkovo, No. 161, 144 (in Russian)

Bronnikova, N. M. 1958b, Trudy Glav. Astron. Obs. Pulkovo, Ser. 2 , 72, 79 (in Russian)

Girardi, L., Bressan, A., Bertelli, G., \& Chiosi, C. 2000, A\&A, 141, 371

Jilinski, E. G., Frolov, V. N., Ananjevskaja, Yu. K., \& Drake, N. A. 2000, Izv. Glav. Astron. Obs. Pulkovo, No. 215, 57 (in Russian) Mermilliod, J. C. 1981, A\&A, 97, 235

Rio, del, G., \& Huestamendia, G. 1988, A\&AS, 73, 425

Sanders, W. 1971, A\&A, 14, 226

Schmidt-Kaler, Th. 1965, Landolt-Bornstein, Neue Ser., 298

Steinlin, U. W. 1968, Z. Astrophys., 69, 276

Trümpler, R. J. 1930, Lick Obs. Bull., 14, 154

Zhukov, L. V. 1966, A Zh., 43, 1107 